\title{
Lumen
}

Selected Proceedings from the Canadian Society for Eighteenth-Century Studies

\section{L’immatérialisme en terre ennemie : la pensée berkeleyenne dans le matérialisme des Lumières}

\section{Sébastien Charles}

Volume 20, 2001

URI : https://id.erudit.org/iderudit/1012302ar

DOI : https://doi.org/10.7202/1012302ar

Aller au sommaire du numéro

Éditeur(s)

Canadian Society for Eighteenth-Century Studies / Société canadienne d'étude du dix-huitième siècle

ISSN

1209-3696 (imprimé)

1927-8284 (numérique)

Découvrir la revue

Citer cet article

Charles, S. (2001). L’immatérialisme en terre ennemie : la pensée berkeleyenne dans le matérialisme des Lumières. Lumen, 20, 49-69.

https://doi.org/10.7202/1012302ar

Copyright (C Canadian Society for Eighteenth-Century Studies / Sociéte canadienne d'étude du dix-huitième siècle, 2001
Ce document est protégé par la loi sur le droit d'auteur. L'utilisation des services d'Érudit (y compris la reproduction) est assujettie à sa politique d'utilisation que vous pouvez consulter en ligne.

https://apropos.erudit.org/fr/usagers/politique-dutilisation/ 


\section{L'immatérialisme en terre ennemie: la pensée berkeleyenne dans le matérialisme des Lumières}

L'opposition conceptuelle entre matérialisme et idéalisme (dont l'immatérialisme sera considéré par les Lumières comme une branche, voire parfois comme un synonyme) date du début du XVIII ${ }^{\mathrm{e}}$ siècle et elle est due à Leibniz qui, dans ses Répliques aux réflexions de Bayle de 1702, écrivait, à propos de sa doctrine de l'harmonie préétablie: «ce qui fait voir que ce qu'il y a de bon dans les hypothèses d'Épicure et de Platon, des plus grands matérialistes et des plus grands idéalistes, se réunit ici ${ }^{1}{ }^{\prime}$. Platon avait bien auparavant opposé, dans le Sophiste (246a), les Fils de la terre aux amis des Formes, ce qui tend à montrer que l'opposition leibnizienne ne se caractérise pas par son originalité - même si l'emploi des termes est nouveau -, et il avait laissé entendre que le défi majeur des Fils de la terre était de tenter le rapatriement des Formes ici-bas. Il est vrai que de tout temps le discours matérialiste a été vu comme réaction plutôt qu'affirmation, d'où son caractère essentiellement clandestin - ce qui lui donne son unité temporelle, unité qui n'interdit pas néanmoins de distinguer entre matérialisme antique et matérialisme moderne. En effet, la matière même dont ces deux matérialismes se réclament est différente, et leur opposition au discours dominant dépend de ce que ce discours lui-même met en valeur: la forme pour les anciens, l'esprit pour les modernes. De même est-il envisageable de distinguer des matérialismes au sein d'une époque déterminée, ainsi pour le XVIII ${ }^{\mathrm{e}}$ siècle qui nous occupe ici.

Si les matérialistes du Siècle des lumières s'entendent sur le primat de la matière sur l'esprit, il reste néanmoins possible, en suivant en cela Olivier Bloch, de distinguer historiquement deux courants dont le milieu du siècle marque la différence: un matérialisme masqué qui s'exprime avant tout durant la première moitié du siècle dans la littérature clandestine et dans quelques ouvrages originaux (ceux de Boulainviller, de Maillet, du curé Meslier) et qui cherche majoritairement à toucher une élite privilégiée à l'aide de références antiques et renaissantes; un matérialisme triomphant, celui de la seconde moitié du siècle, dont les porte-parole osent de plus en plus s'exprimer au grand jour en critiquant 
ouvertement les schèmes spiritualistes et les conventions sociales et politiques (La Mettrie, Diderot, Helvétius, d'Holbach), et qui souhaitent s'adresser au plus grand nombre en tenant compte des découvertes scientifiques de leur temps ${ }^{2}$. Dans cette critique du spiritualisme ne pouvait pas ne pas figurer une attaque en règle de la pensée berkeleyenne qui semblait réduire l'existence des choses extérieures à n'être qu'une simple modification de notre esprit percevant et, par là, nous enfermer dans le solipsisme. L'enjeu de ce texte est de s'interroger sur l'opposition entre matérialisme et immatérialisme pour chercher à comprendre comment des penseurs pour qui la matière est la seule substance à l'œuvre dans l'univers ont pu interpréter une pensée qui lui dénie tout droit à une existence réelle.

Il faut d'abord dire un mot sur le matérialisme du début du siècle et sur ses rapports à l'immatérialisme, car rapports il y eut, aussi étrange que cela puisse paraître. En effet, si la première traduction de Berkeley en langue française (l'Alciphron) ne date que de 1734, les nombreuses recensions critiques des Trois dialogues entre Hylas et Philonous parues en 1713 dans les revues savantes de l'époque avaient contribué à faire de lui l'idéal-type de l'opposant au matérialisme ${ }^{3}$. Mais si certains textes d'auteurs matérialistes en prennent acte, ils ne semblent pas pour autant voir tout l'intérêt de produire une réfutation efficace de cette forme d'idéalisme. L'adversaire reste avant tout Malebranche, non Berkeley, comme on peut s'en rendre compte aisément à la lecture d'un texte de 1714, la Réponse en forme de dissertation à un théologien ${ }^{4}$, du médecin Abraham Gaultier, qui défend un monisme matérialiste d'inspiration biologique. Ce texte a eu une influence importante sur la pensée des Lumières grâce à sa circulation sous forme d'extraits manuscrits sous le titre de Nouvelle philosophie sceptique et de Paritéde la vie et de la mort, avant d'être imprimé sous ce dernier titre en 1770 avec bon nombre de modification $\mathrm{s}^{5}$ (ainsi trouvera-t-on une référence à Berkeley dans le texte de 1770 qui n'existait pas dans celui de 1714). Partisan du phénoménisme (seules les apparences des choses nous sont connues, non les choses elles-mêmes; seuls les effets, non les causes), Gaultier pense qu'il se «trouve en nous un fond d'incertitude insurmontable; puisque les choses nous paroissent diversement, selon la diversité de nos organes ${ }^{6} »$. Le texte de 1770 continuera dans la même veine en ajoutant que "c'est de cette diversité de manieres de voir \& de sentir qu'est née la multitude des systêmes, \& je ne doute point que leurs Auteurs ne fussent pénétrés de la vérité des opinions qu'ils avançoient. Platon voyoit l'Hylée, Épicure les Atomes, Leibniz les Monades; l'Immatérialiste Barklai ne voyoit rien $^{7} . »$ On comprend alors aisément que la philosophie de Malebranche devait lui sembler fort particulière, fondée entièrement sur l'imagination et non sur la raison, d'où sa critique de cette forme de philosophie 
dogmatique qui prétend expliquer l'ordre naturel à partir de quelques principes, sans tenir compte ni de l'expérience ni de la nature, et qui, à vouloir tout expliquer, n'explique en fait absolument rien.

Pourtant, très vite, la critique de l'occasionnalisme va céder la place à celle du scepticisme dont l'immatérialisme sera perçu comme une des formes possibles. C'est le cas par exemple, dès 1718, pour Jean Meslier. Ce dernier a dû connaître Berkeley, puisqu'il a rédigé un commentaire suivi de la préface des CEuvres philosophiques de Fénelon de 1718 dans laquelle le père Tournemine s'en prenait rudement aux conséquences athées de l'immatérialisme, préface qui sera rééditée l'année suivante dans les Mémoires de Trévoux. Pour notre jésuite, "dans ce systême [l'immatérialisme] je crois seulement que je suis, que je pense, ou plûtôt que je rêve, que mes idées n'ont aucune réalité, aucune vérité, ainsi plus de foi humaine, plus de loi, plus de principes, plus de regle de morale ${ }^{8}$. Quant à prouver l'existence de Dieu à partir d'une harmonie mondaine en recourant à cette preuve cosmologique que le Siècle des lumières privilégie, rien de plus absurde si on postule, comme les immatérialistes, l'inexistence du monde extérieur. Meslier, face à ce passage du père Tournemine, est on ne peut plus clair: «ll faut être plus fou que sage pour entrer dans de tels sentiments ${ }^{9} . »$ Par là, il rejoint la critique du pyrrhonisme développée dans son célèbre mémoire qui associe le refus réel de l'existence des corps extérieurs à une pure et simple folie et qui montre que les pyrrhoniens conséquents se jouent et ne pensent pas réellement ce qu'ils avancent: «le pyrrhonisme ne va pas jusques à douter de telles choses, ainsi on peut dire qu'il est plus imaginaire que reel, et que c'est plutot un jeu d'esprit qu'une veritable persuasion de l'âme ${ }^{10}{ }^{\prime}$.

Les choses auraient pu en rester là si l'immatérialisme avait continué à n'être simplement qu'un opposant fantoche et peu crédible contre lequel la raillerie est la meilleure des réfutations. Mais, durant les trente années qui suivent, l'influence de Berkeley ne va cesser de s'affirmer. Nous ne retracerons ici que rapidement les grandes étapes de cette affirmation. Les années 1720-1735 sont encore marquées par une méconnaissance et un travestissement indéniables des positions réelles de l'immatérialisme berkeleyen. Le père Buffier, reprenant à son compte la problématique de l'existence des corps extérieurs qui secoue ce début de siècle dans son Traité des premieres véritez de 1724, mentionne l'existence de «Philosophes de ce tems [qui] n'ont pas daigné reconnoître ni admettre d'autres genres de vérité \& d'évidence [que celle du sentiment intime]». D'ailleurs, nous apprend-il, «c'est ce qu' un philosophe Anglois n'a point fait difficulté de publier ${ }^{11}{ }^{»}$. Ce philosophe anglais, l'année suivante dans les Élémens de métaphisique, devient écossais et auteur d'un ouvrage destiné à "prouver qu'il n'avoit aucune évidence de l'éxistence d'aucun être que lui: \& encore de lui, en tant qu'esprit; mais non de lui, 
en tant que corps: n'aïant aucune démonstration véritable de l'éxistence $\mathrm{d}^{\prime}$ aucun $\operatorname{corps}^{12}{ }^{»}$. Jean-Pierre de Crousaz, tout aussi énigmatique sur la paternité réelle de l'auteur de la théorie immatérialiste, discerne mieux son enjeu. Aussi peut-il écrire en 1733 dans son Examen du pyrrhonisme ancien $\mathcal{E}$ moderne qu'

un Auteur moderne prétend renverser le Pyrrhonisme, en niant l'Existence des Corps, \& en n'admettant que celle des Esprits. S'il a dessein d'imposer par-là au reste des hommes, \& qu'il espère d'y réüssir, il en a bien mauvaise opinion; \& s'il pense comme il parle, il ne se donne pas une grande Idée de son bon-sens, \& il faut qu'il suppose le Cerveau des autres hommes autant renversé que le sien l'est effectivement ${ }^{13}$.

La même année, dans ses Pensées critiques sur les mathématiques, François Cartaud de la Villate mentionne l'existence d'une secte, "celle des Immatérialistes, qui a beaucoup de partisans en Angleterre; quoiqu'ils pensent que l'existence des Corps n'est pas bien démontrée, \& que le plus grand nombre d'entr'eux affirment positivement qu'il n'y en a point, que tout est idéal, ou n'existe qu'en idée ${ }^{14}{ }^{\prime}$. Le sort de Berkeley, dans ces années 1720-1735, est similaire à celui de ces égoïstes, cette «secte fantôme» selon l'heureuse expression de Jean-Robert Armogathe ${ }^{15}$, dont tout le monde parle sans pouvoir pour autant ni en définir avec précision les concepts ni en nommer avec certitude les principaux représentants - ce qui explique peut-être un amalgame rapide entre égoïsme et immatérialisme et l'émergence de la figure de Berkeley comme chef de file incontesté du mouvement.

C'est sans doute la traduction de l'Alciphron en 1734 qui va permettre une connaissance plus approfondie des thèmes berkeleyens, et la censure dont l'ouvrage a été l'objet lui a accordé un certain statut. Mais c'est plus certainement le rôle de Voltaire qui a été ici capital, comme nous l'avons montré ailleurs $^{16}$. En soulignant les erreurs de lecture de Desfontaines en réponse à la Voltairomanie de ce dernier, il permettait de rendre à Berkeley un visage authentique. En critiquant la théorie optique de Newton à partir de sa lecture de l'Essai pour une nouvelle théorie de la vision, il donnait à Berkeley, en tant qu'opposant victorieux, une stature non négligeable. Mais le mal était fait. Saint-Hyacinthe, sans doute l'un des premiers à lire Berkeley dans le texte et à ne pas se contenter des recensions partiales qui ont été faites de ses ouvrages, mêle encore en 1743 égoïsme et immatérialisme, même s'il disqualifie l'interprétation de Tournemine qui en faisait un athée. Chose importante, il avoue que cette théorie est «une des plus difficiles à refuter, si mème on le peut ${ }^{17}$ ». Or c'est là le leitmotiv de Diderot à l'égard de l'immatérialisme. 
La position de Diderot est sans nul doute la plus élaborée, d'une part parce que son intérêt pour l'immatérialisme est constant et d'autre part parce qu'il est le seul penseur matérialiste à avoir compris toute la nécessité d'une réfutation efficace de cette doctrine. Témoin ce Projet $d^{\prime} u n$ traité qui date certainement de la fin de sa vie où, dans la partie «Les sciences», on peut lire: "La métaphysique. Berkeley ${ }^{18}$." Il faut ici comprendre que, pour Diderot, une métaphysique qui n'aurait pas mis en question ses fondements à travers une confrontation critique avec Berkeley ne vaudrait pas une heure de peine. C'est ce qu'il tente de faire comprendre à Hemsterhuis en 1773-1774, quand il annote une copie de sa Lettre sur l'homme et ses rapports, commentaires dont le penseur hollandais ne tiendra aucun compte dans ses ouvrages suivants ${ }^{19}$. Là où Hemsterhuis, un peu vite, conclut de la sensation d'un objet à son existence réelle hors du sujet, Diderot note: «Voilà ce que Berkeley ne vous passerait jamais. Je suis moins difficile que lui, quoique je ne sache aucune solide réponse à son sophisme contre l'existence de quoi que ce soit hors de nous. L'esprit humain en est humilié, mais il n'en est ni persuadé ni convaincu ${ }^{20}$.» Cette note manuscrite a le mérite de montrer clairement la position de Diderot face à l'immatérialisme: acceptation théorique d'une doctrine considérée comme irréfutable; condamnation pratique des effets qui pourraient s'ensuivre.

Quand Diderot en est-il venu à s'intéresser à l'immatérialisme? Le nom de Berkeley est présent dans son œuvre dès le début de 1747, car il figure expressément dans la Promenade du sceptique, mais le poids de l'œuvre s'y fait également sentir, puisqu'un personnage se dénomme Alciphron et qu'un égoïste se permet d'intervenir dans les débats philosophiques. Reste à dater cette influence. Pour Jacques Chouillet, «Diderot est venu à Berkeley par Condillac ${ }^{21}{ }$ » et il n'aurait pris connaissance de l'idéalisme que par le sensualisme de ce dernier. On trouve la même idée chez Jean Deprun ${ }^{22}$, qui rappelle néanmoins aussi une lecture possible de Saint-Hyacinthe, ou chez Ursula Winter ${ }^{23}$. Nous voudrions ici renverser la filiation en apportant un élément nouveau, sans néanmoins nier toute l'importance de la lecture de l'Essai sur l'origine des connaissances humaines paru en 1746. Si, pour la majorité des commentateurs, Condillac précède Berkeley dans l'histoire de la formation intellectuelle de Diderot, c'est parce qu'ils s'accordent sur une date pour la traduction par JeanPaul de Gua de Malves des Trois dialogues entre Hylas et Philonous, à savoir 1750. Or, si l'on en croit Saverien qui la cite explicitement, la première édition en langue française date de 1744 (le lieu de la publication est manquant $)^{24}$. Ce fait confirme ce qu'évoquait déjà la Bibliographie universelle ancienne et moderne qui, à l'article "Gua de Malves", mentionnait les deux dates de 1744 et de $1750^{25}$. De plus, si l'on s'en tient exclusivement à la date de 1750, comment expliquer la référence que Diderot fait 
à la traduction française des Dialogues dans la Lettre sur les aveugles de 1749 , qui dit textuellement qu'il serait bon que Condillac les lise, ce qui laisse supposer qu'il n'en avait pas réellement pris connaissance auparavant ${ }^{26}$ ?

La proximité de ces dates rend donc troublante l'hypothèse d'une première publication en 1750. Malgré tout, nous n'en disconvenons pas, cette édition a sans nul doute connu une diffusion très restreinte, puisqu'à ce jour nous n'avons pu mettre la main sur un seul exemplaire. Toutefois, nous pouvons en admettre l'existence. Alors, puisque Diderot connaissait Gua de Malves dès 1745 ou $1746^{27}$, comment aurait-il pu l'ignorer? De plus, le projet confié à Gua de Malves à cette date par les libraires parisiens, projet que Diderot et D'Alembert reprendront seuls à leur compte et qui donnera naissance à l'Encyclopédie, avait pour but la traduction de la Cyclopædia de Chambers, dont plusieurs articles explicitaient la pensée de Berkeley ${ }^{28}$. Dès lors, il faut plutôt penser que c'est Berkeley qui a mené Diderot à Condillac, et non l'inverse, et que l'immatérialisme était peut-être un sujet de conversation évoqué à l'hôtel du Panier fleuri où il dînait chaque semaine en compagnie de Condillac et de Rousseau, conversation à laquelle Turgot et D'Alembert auraient pu prendre part ${ }^{29}$. Sinon, comment expliquer, sans une possible connaissance de Berkeley à cette date, la lecture immatérialiste que Diderot fait de la phrase introductive de l'Essai de Condillac qui annonce que, «Soit que nous nous élevions, pour parler métaphoriquement, jusque dans les cieux, soit que nous descendions dans les abîmes, nous ne sortons point de nous-mêmes, et ce n'est jamais que notre propre pensée que nous apercevons $\mathrm{s}^{30}$ », proposition qu'il attribuera jusqu'à la fin de sa vie alternativement à Condillac et à Berkeley?

Venons-en maintenant plus en détail à la lecture diderotienne de Berkeley. Le nom de l'évêque de Cloyne apparaît donc en 1747, dans le discours préliminaire de la Promenade du sceptique, à une époque où Diderot s'interroge sur les positions théoriques et pratiques du pyrrhonisme. La liberté de penser de Berkeley est mentionnée à l'égale de celle des Bayle, Montaigne, Voltaire, Woolston, Swift ou Montesquieu, souvenir de la condamnation par la censure de l'Alciphron en 1734. En outre, dans la Promenade, la position solipsiste ou égoïste est rappelée: dans l'allée des marronniers surgit un "champion singulier» qui "soutient qu'il est seul au monde». Les partisans de cette opinion

admettent l'existence d'un seul être; mais cet être pensant, c'est eux-mêmes: comme tout ce qui se passe en nous n'est qu'impression, ils nient qu'il y ait autre chose qu'eux et ces impressions; ainsi ils sont tout à la fois l'amant et la maîtresse, le père et l'enfant, le lit de fleurs et celui qui le foule ${ }^{31}$. 
Et Diderot d'en rester à une boutade. Le ton est bien différent deux ans plus tard, dans la Lettre sur les aveugles, quand il s'agit pour Diderot de définir l'idéalisme, cette doctrine dont les sectateurs «n'ayant conscience que de leur existence et des sensations qui se succèdent au-dedans d'eux-mêmes, n'admettent pas autre chose», et dont Berkeley a exposé avec «franchise et clarté» les principes. Philosophes aussi aveugles sur le plan théorique que Saunderson l'est réellement, car ils n'ont pas besoin des corps extérieurs pour en définir toutes les propriétés; et philosophes dangereux, car leur système absurde, «à la honte de l'esprit humain et de la philosophie, est le plus difficile à combattre ${ }^{32} \gg$. Diderot engage Condillac à le faire, sur la base d'une similitude entre ses principes et ceux de Berkeley, tout comme le fera un an plus tard Raynal ${ }^{33}$. Il est sûr que le Traité des sensations de ce dernier ne sera pas, pour Diderot, une réponse efficace à l'immatérialisme, puisqu'il continuera à souligner par la suite toute la portée de la philosophie de Berkeley. C'est sans doute D'Alembert qui formule le plus justement l'irréfutabilité de l'immatérialisme dans l'article «Égoïstes» du tome V de l'Encyclopédie:

L'Égoïsme est le Pyrrhonisme poussé aussi loin qu'il peut aller. Berkley, parmi les modernes, a fait tous ses efforts pour l'établir. Les égoïstes sont en même tems les plus extravagans des Philosophes, \& les plus difficiles à convaincre; car comment prouver l'existence des objets, si ce n'est par nos sensations? \& comment employer cette preuve contre ceux qui croyent que nos sensations ne supposent point nécessairement qu'il y ait quelque chose hors de nous? Par quel moyen les fera-t-on passer de l'existence de la sensation à celle de $\mathrm{l}^{\prime}$ objet $^{34}$ ?

D'Alembert est assez fin pour reconnaître que les matérialistes euxmêmes savent pertinemment que la sensation subjective ne renseigne en rien sur la réalité même de l'objet, car elle nous fournit uniquement une connaissance de ce qui se donne à percevoir. C'est pourquoi il a pu écrire dans le tome précédent de l'Encyclopédie, à l'article «Corps», "que les partisans même de la matiere nient qu'il puisse y avoir aucune connexion entre [l'existence des corps hors de notre esprit $]$ \& nos idées ${ }^{35}{ }^{\prime}$. C'est bien là le problème qui se pose à Diderot, et en général à toute philosophie qui se fonde sur l'axiome scolastique qui énonce que nihil est in intellectu quod non fuerit prius in sensu. Car le seul moyen de répondre définitivement à l'immatérialisme, pour Diderot, suppose une objectivité pure: «Il faudroit pour le confondre, que je puisse sortir de la nature, l'en tirer, et raisonner de quelque point hors de lui \& de moi, ce qui est impossible ${ }^{36}$.» C'est pourquoi Diderot écrit à Vialley en juillet 1766 qu'on n'a pas encore bien répondu à Berkeley, mais sans lui confier pour autant que cela lui paraît impossible ${ }^{37}$. En décembre de la même année, toujours à travers sa correspondance, mais avec Falconet cette 
fois, il découvre toute la force du matérialisme à partir de l'immatérialisme même. Si nous ne sortons jamais de nous-mêmes, si tout se passe en nous, alors «nous sommes l'univers entier. Vrai ou faux, j'aime ce système qui $\mathrm{m}^{\prime}$ identifie avec tout ce qui $\mathrm{m}^{\prime}$ est $\operatorname{cher}^{38} .{ }^{\circ}$ Cette hypothèse apparaît de nouveau trois ans plus tard dans ce chef-d'œuvre philosophique de Diderot qu'est le Rêve de D'Alembert, toujours en rapport avec celle de Berkeley. Faisant de l'homme un être doué de sensibilité et de mémoire, matériel de part en part, Diderot affirme à un D'Alembert imaginaire que ce qui donne à son système toute sa force, c'est qu'il «est sujet à la même difficulté insurmontable que Berkeley a proposée contre l'existence des $\operatorname{corps}^{39}{ }^{\prime \prime}$, à savoir l'impossibilité de trouver une position objective, ce qui le rend également irréfutable.

Diderot a dès lors compris que matérialisme et idéalisme sont deux positions métaphysiques indémontrables sur lesquelles peuvent se greffer des systèmes vraisemblables mais non certains. Le bel ordre des raisons cartésien est rompu, car l'hypothèse a remplacé l'évidence au fondement de la chaîne des découvertes. Matérialisme et immatérialisme se rejoignent néanmoins sur un point: il est strictement impossible de prouver l'existence des corps extérieurs. Comme l'écrit encore Diderot deux ans avant sa mort, dans la deuxième édition de son Essai sur les règnes de Claude et de Néron, "Berkeley attend encore une réponse $^{40}{ }_{»}$. Quelle réponse, si elle ne peut être théorique? La seule opposition réelle à l'idéalisme est pratique, c'est celle qui consiste à considérer cette position comme la solution du pauvre, vrai sophisme paresseux qui dénie à l'action toute validité. Diderot sait bien qu'un pyrrhonien ne peut être conséquent, et c'est là le talon d'Achille de sa doctrine; car pourquoi chercherait-il à diffuser ses idées s'il n'était intimement persuadé que de sa seule existence? C'est ce qu'a bien compris également Le Guay de Prémontval, qui réfute l'immatérialisme par l'ironie:

Rien ne me paraitroit plus plaisant que le sistème d'un égoîste dédié à un mècenes [sic] dont il brigueroit la faveur; si pourtant le livre en soi n'étoit déjà aussi plaisant qu'il pût l'être. Prouver à d'autres que l'on est seul! Je crois que la dédicace commenceroit ainsi: «Monseigneur!... De toutes les apparences dont j'éprouve les sensations, il n'en est point pour qui j'ai une vénération plus profonde, que pour votre Excellence.» Ensuite il se loueroit infiniment de cette sensation; des obligations qu'il reconnoît lui avoir, de la généreuse protection qu'il en attend. Il vanteroit le mérite \& les vertus qu'il y admire, \& débiteroit toutes les impertinences qu'on débite à des réalités. Laissons ce fou... ${ }^{41}$

Moins railleur, car ayant l'esprit plus philosophique, Diderot voit bien lui aussi l'impossibilité réelle d'une doctrine théoriquement irréfutable. 
Comme Diogène répondait à Zénon qui niait l'existence du mouvement par la marche, Diderot répond à Berkeley par l'action: «l'homme un \& vrai n'a point deux philosophies, l'une de cabinet \& l'autre de société; il n'établit point dans la spéculation des principes qu'il sera forcé d'oublier dans la pratique ${ }^{42} »$. Par là, il rejoint une intuition de jeunesse à l'œuvre dans les notes de sa traduction de l'Essai sur le mérite et la vertu de Shaftesbury: "À quoi bon me prescrire des règles de conduite, dira peut-être un pyrrhonien, si je ne suis pas sûr de la succession de mon existence? Cependant, on agit, on se pourvoit, comme si rien n'était plus vrai: le pyrrhonien même laisse ces subtilités à la porte de l'école, et suit le train commun ${ }^{43}$.» Mais ce pyrrhonien doit être distingué du sceptique qui reconnaît les limites de toute spéculation et dont la pratique assume ces limites. Dans une certaine tradition sceptique, qu' un Montaigne, par exemple, nomme pyrrhonisme, cette séparation de la théorie et de la pratique est même envisagée positivement, en tant que base pour la recherche de l'ataraxie, et non seulement négativement, comme conséquence néfaste des excès du savoir théorique. Diderot partage cette vue dans l'article «Pyrrhonienne» de l'Encyclopédie où il vante l'ignorance humaine, en tant qu'elle ne se réduit pas à une incertitude dogmatique. Car c'est cette forme de scepticisme que Diderot combat, qui consiste à limiter les prétentions du savoir dans les domaines où celui-ci ne peut plus statuer; et c'est en ce sens que Diderot dit qu'il n'écoutera plus l'homme qui nie l'existence des corps, et qu'il ne cherchera pas à détruire chez cet homme des opinions qui ne sont pas vraiment les siennes. L'adversaire ici n'est pas le vrai sceptique, ou le pyrrhonien de Montaigne, mais l'égoïste, le solipsiste, devenu fou à force de spéculations oiseuses, et que l'on ne peut pourtant pas réfuter par l'argumentation:

Que dirai-je à celui qui prétendant que, quoi qu'il voye, quoi qu'il touche, qu'il entende, qu'il apperçoive, ce n'est pourtant jamais que sa sensation qu'il apperçoit: qu'il pourroit avoir été organisé de maniere que tout se passât en lui, comme il s'y passe, sans qu'il y ait rien au-dehors, \& que peut-être il est le seul être qui soit? Je sentiroi tout-à-coup l'absurdité \& la profondeur de ce paradoxe; \& je me garderai bien de perdre mon tems à détruire dans un homme une opinion qu'il n'a pas, \& à qui je n'ai rien à opposer de plus clair que ce qu'il nie ${ }^{44}$.

Le type même de l'adversaire ainsi défini, c'est Louis de Beausobre, auteur d'un Pyrrhonisme du sage, qui défend les positions que Diderot condamne. Dans cet ouvrage paru en 1754 et réédité l'année suivante sous un titre différent, Beausobre remet en question le sensualisme en dénonçant les acquis de la perception sensible, et le matérialisme en 
prêchant l'idéalisme, sans pour autant se décider en faveur d'un système $^{45}$, faisant du doute le siège théorique de l'homme sage:

Nous ne sçaurions donc nous flater de connoître quelque chose avec certitude. Il faut se contenter d'entrevoir confusément au travers d'un rideau, \& de conjecturer ce qui pourroit bien y être caché. Les conjectures prennent la place de la vérité; \& au défaut du certain, nous jouissons du probable: c'est une foible lumiére, mais une plus grande nous éblouiroit ${ }^{46}$.

L'article «Doute», qui a peut-être Diderot pour auteur ${ }^{47}$, a pour but explicite de remettre en question cette forme de pyrrhonisme. Douter, $c^{\prime}$ est ne pas pouvoir trancher; or rares sont les matières où il ne soit pas possible d'opter en faveur d'une opinion. Cela implique que le pyrrhonisme ne peut être une doctrine conséquente. «Heureusement pour les Pyrrhoniens, l'instinct supplée avec usure à ce qui leur manque du côté de la conviction, ou plutôt il corrige l'extravagance de leur doute ${ }^{48}$.» Mais un pyrrhonien ne pourrait-il pas répliquer que la probabilité lui suffit pour se déterminer et que, s'il ne connaît pas l'essence des choses, les apparences sont un gage suffisant pour agir? C'est ainsi que Huet semblait, à l'avance, répondre aux critiques de Diderot:

Lors qu'il s'agit de conduire sa vie, de s'acquitter de ses devoirs, nous cessons d'être Philosophes, d'être contrarians, douteux, incertains; nous devenons idiots, simples, credules; nous appellons les choses par leurs noms; nous reprenons nos mœurs \& nôtre Esprit; nous conformons nos mœurs aux mœurs des autres hommes, à leurs coûtumes, à leurs loix. Moi qui doutois tantôt si j'étois, s'il y avoit d'autres hommes, je bannis maintenant toutes ces pensées; \& comme étant assuré que je suis, \& que les autres hommes sont, je mange, je bois, je marche, je vais voir mes Amis, je les salue, je les entretiens, $j$ 'affirme, je nie, $j$ ' assure que cela est vrai, que cela est faux. Car, comme dit Ciceron, il y a grande difference entre la subtilité avec laquelle on recherche la Vérité dans la dispute; $\mathcal{E}$ celle avec laquelle on ajuste son discours à l'opinion commune $e^{49}$.

Diderot pousse alors le sceptique dans ses derniers retranchements. Suivre l'opinion commune, comme Huet le recommande, c'est affirmer un principe, et donc rompre avec le scepticisme. "Sceptiques dans leur façon de penser, comment peuvent-ils être dogmatiques dans leur maniere d'agir? Ce seul point qu'ils accordent, est un écueil où viennent se briser toutes leurs vaines subtilités ${ }^{50} . »$ Si la pratique ruine la théorie sceptique, c'est à la fois parce qu'elle force le pyrrhonien à renoncer à son doute quand il lui faut agir, mais aussi parce qu'elle suppose une forme d'évidence, l'action ne pouvant être sans délibération ni théorisation. Cette réfutation, qui vaut contre le pyrrhonisme, vaut également 
contre Berkeley qui n'est, aux yeux de Diderot, que l'un des sectateurs modernes de cette philosophie absurde parce qu'intenable et qui n'a comme conséquence que de ruiner le lien social.

C'est la leçon de Diderot, qui est celle du matérialisme des Lumières dans son ensemble. Ainsi Helvétius, dans une note du chapitre I du discours premier de De l'esprit, voit-il dans l'existence des corps extérieurs une grande certitude, mais non une vérité absolue. Pour lui, le problème de l'existence des corps extérieurs est aussi difficilement résoluble que celui de la définition de la nature spirituelle ou matérielle de l'âme. Une évidence de type cartésien, sur ces deux points, est impossible à dégager. La seule certitude qu'Helvétius concède, c'est celle du sentiment intime de notre existence propre. Mais pour les corps extérieurs, la chose en va tout autrement:

Dieu, par sa toute-puissance, ne peut-il pas faire sur nos sens les mêmes impressions qu'y exciteroit la présence des objets? Or, si Dieu le peut, comment assurer qu'il ne fasse pas à cet égard usage de son pouvoir, et que tout l'univers ne soit un pur phénomene? D'ailleurs, si dans les rêves nous sommes affectés des mêmes sensations que nous éprouverions à la présence des objets, comment prouver que notre vie n'est pas un long rêve ${ }^{51}$ ?

Vérité et certitude étant équivalentes pour Helvétius, ce qui n'est pas assuré (l'existence des corps extérieurs) ne saurait détenir le statut de l'évidence et devra alors se rabattre sur celui de la probabilité, "probabilité qui sans doute est très grande, et qui, dans la conduite, équivaut à l'évidence, mais qui n'est cependant qu'une probabilité ${ }^{52} \gg$. D'où le vœu pieux d'Helvétius en faveur d'un

homme de génie qui se chargeroit de construire des tables physiques, métaphysiques, morales et politiques, où seroient marqués avec précision tous les divers degrés de probabilité, et par conséquent de croyance, qu'on doit assigner à chaque opinion ${ }^{53}$,

vœu que Jean-Claude de La Métherie tentera de satisfaire ${ }^{54}$. Helvétius se contente d'en tracer les fondements et de montrer que l'existence des corps extérieurs obtiendrait le premier degré de certitude dans les tables physiques, tout comme celles de Rome ou de Londres obtiendraient le même degré de certitude dans les tables morales, cette évaluation des probabilités ayant pour but de faire progresser les hommes dans l'ordre du savoir. Si le texte d'Helvétius ne mentionne pas explicitement Berkeley, ses contemporains ne furent pas dupes. Ainsi Barruel, dans un passage de ses Helviennes où il raille ces penseurs qui ne savent pas définir l'essence même de la matière, dira d'Helvétius «qu'un philoso- 
phe anglois l'avoit entraîné ce jour-là dans son opinion ${ }^{55}$ ». Mais que l'existence des corps soit ou non une certitude, Helvétius n'en pensait pas moins possible l'élaboration d'une science des phénomènes.

D'Holbach, qui se pose le même problème, y apporte une réponse équivalente. En matérialiste qui se respecte, il est obligé de concéder que "s'il n'est point permis à des êtres raisonnables de douter des vérités qui leur sont démontrées par l'expérience de tous les siècles, il leur est permis d'ignorer et de douter de la réalité des objets qu'aucun de leurs sens ne leur a jamais fait connaître ${ }^{56}$ ", scepticisme qui vaut bien sûr dans son esprit contre les êtres spirituels, qui nous sont imperceptibles, mais qui atteint aussi la matière dont nous ne percevons que les modifications et non la substance. En fait, il s'agit d'un pyrrhonisme affiché, qui n'est pas réel, puisque le rôle de la matière comme condition causale de la sensation est sous-entendu. Un réel sceptique aurait dû dire, comme le fit l'abbé Galiani dans une lettre à Mme d'Épinay en date du 30 juin 1770, qu'«au fond nous ne connaissons pas assez la nature pour en former le système $^{57}$ ». Mais, dans l'ensemble, comme l'écrit à juste titre Yvon Belaval, «le scepticisme des Lumières n'aura souvent été que l'excuse du dogmatisme honteux de ses incertitudes ${ }^{58} »$. Les lecteurs contemporains ne s'y sont pas trompés, voyant bien que le scepticisme invoqué n'était que de façade et que le matérialisme ne pouvait se prévaloir de n'être pas, lui aussi, systématique alors même qu'il prétendait définir le réel comme entité globalisante. Dom Deschamps est là catégorique:

Qu'est-ce qu'un système général physique sinon un système métaphysique? Le métaphysique peut-il être autre chose que la généralité du physique? [...] Le système d'un astronome, d'un naturaliste, d'un médecin et de tout physicien qui se borne à sa science est physique en ce qu'il est particulier. [...] Mais le vôtre embrasse le système général des êtres pour prouver qu'il n'y a pas de Dieu et que l'homme n'est pas d'une autre nature que le reste des êtres; et c'est par là qu'il est métaphysique quoique sans principe métaphysique ${ }^{59}$.

Confirmation d'une telle entreprise est donnée par le Système de la nature, ouvrage qui résume à lui seul les tendances matérialistes du siècle et qui se présente comme le catéchisme du matérialisme explicitant l'ensemble du réel, car, «l'affirmation centrale de d'Holbach, ce n'est pas seulement l'unité de la matière, c'est avec elle l'unité de l'univers entier, c'est le monisme ${ }^{60}{ }^{\prime}$. D'Holbach est manifestement séduit par l'idée de l'unité de substance, qu'il associe à celle de nécessité ${ }^{61}$. Reste que la possibilité d'un monisme spiritualiste ne peut être écartée du revers de la main, et $\mathrm{d}^{\prime}$ Holbach en est conscient. Sur cette question du monisme, c'est entre Spinoza et Berkeley qu'il faut choisir. Ou bien, avec le premier, on accepte une réduction du réel au matériel, ou bien, avec le second, on 
$s^{\prime}$ accorde «à nier l'existence de l'univers matériel ${ }^{62}{ }^{\text {». }} C^{\prime}$ 'est le chapitre $X$ de la première partie du Système de la nature qui va trancher le nœud gordien du monisme. Pressé de réfuter l'innéisme pour prouver que nos idées sont issues de sensations exercées sur nous par des objets extérieurs, d'Holbach doit avant tout réfuter la doctrine de ceux qui pensent que l'univers entier ne joue aucun rôle dans le processus sensoriel. D'où l'intervention, à ce niveau précis de son analyse, de Berkeley:

Que dirons-nous d'un Berkeley, qui s'efforce de nous prouver que tout dans ce monde n'est qu'une illusion chimérique, que l'univers entier n'existe que dans nous-mêmes et dans notre imagination, et qui rend l'existence de toutes choses problématique à l'aide de sophismes insolubles pour tous ceux qui soutiennent la spiritualité de l'âme ${ }^{63}$ ?

Que lui dire en effet $s i$, avec Diderot ${ }^{64}$, il faut convenir que «le plus extravagant des systèmes (celui de Berkeley) est le plus difficile à combattre. Abyssus abyssum invocat ${ }^{65} »$ ? Et d'Holbach de rappeler la parenté, désormais incontournable, qui unit Malebranche à Berkeley, et d'exposer la doctrine de la vision en Dieu qu'il ridiculise en expliquant que, poussée à son extrême, cette doctrine doit concéder que c'est en Dieu que l'homme voit les hérésies et les schismes, et que c'est donc en Dieu que

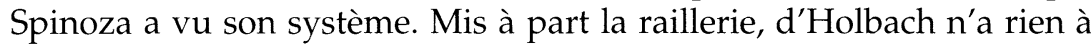
répondre aux partisans du solipsisme en qui il discerne des disciples de l'occasionalisme et de l'immatérialisme, si ce n'est que l'hypothèse de la matière paraît hautement plus vraisemblable: «Prétendre que l'homme, sans le secours des objets extérieurs et des sens, peut avoir des idées de l'univers, c'est dire qu'un aveugle né peut avoir l'idée vraie d'un tableau représentant quelque fait dont jamais il n'aurait entendu parler ${ }^{66} . »$ Mais les songes ne prouvent-ils pas, comme le soupçonnaient déjà certains idéalistes, que l'âme a la faculté de produire des idées sans être affectée par des corps matériels? Il ne faut voir là qu'une ineptie, nous dit $\mathrm{d}^{\prime} \mathrm{Holbach}$, puisque les idées qui parsèment nos rêves sont déjà celles de la veille, et que tout se réduit donc au matériel seul. Penser autrement, c'est être de mauvaise foi.

C'est ce que montre l'autre texte de d'Holbach consacré à Berkeley, où l'on peut lire que "si l'on est de bonne foi, l'on ne pourra se faire illusion au point de douter de sa propre existence et de celle d'êtres qui nous ressemblent, dont nous sommes entourés, sur lesquels nos actions influent, et qui réagissent sur nous, selon la manière dont ils sont affectés par nos propres actions ${ }^{67}$ ». Berkeley, ce «sceptique extravagant», est donc un penseur de mauvaise foi. Penser qu'il n'existe rien de réel en dehors de nous et que toutes nos idées sont un produit de notre imagination et non des effets indiquant l'existence d'un monde extérieur, cela 
peut peut-être affecter la philosophie théorique, mais non la pratique. En effet,

cette hypothèse subtile et bizarre n'exclueroit pas la morale; si, comme ce philosophe le suppose, tout ce que nous voyons dans le monde n'est qu'une illusion, un rêve continuel. En suivant les préceptes de la morale, les hommes se procureront au moins des rêves suivis, agréables, utiles à leur repos, conformes à leur bien-être durant le temps de leur sommeil en ce monde, et les individus qui rêveront ne se troubleront point les uns les autres par des songes funestes ${ }^{68}$.

Bref, si, en théorie, l'idéalisme a un quelconque intérêt en ce qu'il permet de dénoncer les excès de l'imagination philosophique ( $d$ 'Holbach n'hésitant pas à retourner les armes de Malebranche contre lui-même en se servant de sa théorie de l'imagination pour condamner son occasionnalisme), en pratique, il n'est d'aucune utilité et n'a aucune influence puisque, à concéder même qu'il puisse avoir une certaine pertinence, il ne mettrait résolument pas en question les principes moraux que le matérialisme holbachique s'efforce de définir.

Autre trace d'un intérêt à réfuter l'immatérialisme, mais dans la littérature clandestine cette fois, le Jordanus Brunus Redivivus manuscrit rédigé en français malgré ce que son titre peut laisser penser et que l'on peut dater des années 1760-1770 — s'intéresse aussi au problème de l'existence des corps et propose de renverser les conclusions idéalistes ${ }^{69}$. Célébrant dès son avertissement l'expérience comme «une des plus belles facultés de l'homme» (f. 1), vue comme le sol fécond d'où sont sorties les différentes sciences, l'auteur anonyme de ce manuscrit s'attache d'emblée à montrer comment la religion est opposée par nature au développement de cette faculté et comment elle en est venue à châtier ces novateurs qui constituent au XVIII ${ }^{\mathrm{e}}$ siècle la galerie des martyrs de la libre pensée: Cardan, Ramus, Galilée, Bruno et Vanini. Dans le premier chapitre, l'auteur du Jordanus Brunus Redivivus justifie le titre de son traité puisqu'il fait revivre l'hypothèse brunienne de la pluralité des mondes pour mieux amener son lecteur à mettre en doute les certitudes dogmatiques concernant l'unicité du monde qui pourraient l'habiter. Dans le deuxième, il fait de la douleur et du plaisir les seuls critères de la vérité, avouant ainsi que nos connaissances se restreignent «dans un cercle qui n'embrasse rien au-delà de ce qui est utile à notre propre conservation ${ }^{70}$ ». Dès lors, ce qui dépasse ces bornes sensibles que la nature nous a prescrites est superfétatoire. Ainsi, la métaphysique, qui prétend les franchir, n'est qu' une science oiseuse; quant à la question de l'existence de Dieu, elle n'est d'aucun intérêt. Pour ceux qui souhaiteraient malgré tout nous amener à l'évoquer et qui iraient jusqu'à nous forcer à accepter leurs positions théologiques par la contrainte physique, 
ils ne feraient, en recourant à l'usage de la douleur à notre endroit, que devenir automatiquement les ennemis mêmes de notre nature et nous aurions le devoir, et surtout le droit, de nous opposer à leurs visées. Le meilleur moyen de se défendre face à de tels individus ne peut être alors que de détruire rationnellement leur position en en sapant les présupposés. Le troisième chapitre va poser en ce sens le problème de l'existence de Dieu - ou plutôt de son inexistence, car le matérialisme de l'auteur est ici censé tout expliquer à partir d'un œuf originel et des lois physiques de l'attraction et de la gravitation -, en proposant une généalogie historique et psychologique originale de l'invention de cet Etre suprême. La fin du chapitre roule sur des notions d'ordre théologique comme celle de l'infinité et de l'indépendance (la transcendance, dirait-on aujourd'hui) divines, de la relation que Dieu entretient avec l'homme et le diable, de l'incarnation et de la trinité - le tout étant bien sûr de montrer l'absurdité de tels concepts. Le quatrième chapitre s'attaque au dogme de l'immutabilité divine en s'appuyant sur des passages du texte biblique qui mettent en scène un Dieu changeant et capable de modifier ses décrets comme bon lui semble. Le cinquième chapitre, qui est aussi le dernier, fait du mal mondain le point d'achoppement de toute démonstration de l'existence de Dieu. D'où la conclusion définitive du Jordanus Brunus Redivivus: «un Être tel que Dieu est un Être impossible ${ }^{71}$ ». C'est dans le troisième chapitre, intitulé «De l'existence de Dieu», que le manuscrit en vient à évoquer la doctrine immatérialiste. Après avoir montré qu'une seule substance est présente dans l'univers, la question se pose, comme chez d'Holbach, d'en déterminer la nature. Or, si l'on se refuse à voir que la matière seule peut remplir ce rôle, dans quelles absurdités ne tombe-t-on pas... Et pourtant, certains philosophes, «fermant les yeux sur les propriétés sans nombre dont la matière est fournie, sur le mouvement dont elle est douée, sur les productions variées qui sont le résultat de ses mouvemens divers, sur la solidité et la consistence de ses parties», ont «soutenu qu'elle n'existoit pas $^{72}{ }$. Comment répondre à ces penseurs qui ne voient dans les corps extérieurs que des idées? Ne peut-on pas leur dire que nos sens témoignent en faveur de l'existence d'un monde hors de nous, et que notre action le modifie? «Il est donc impossible que dans toutes ces opérations le corps agent et le corps patient n'ayent point une existence réelle, puis qu'ils sentent réellement leur action réciproque les uns sur les autres ${ }^{73}$.» Mais c'est supposer ce qui est en question et les philosophes immatérialistes ne concèdent ni l'existence d'organes sentants ni celle d'objets sentis. De plus, cette théorie philosophique doit être prise sérieusement en compte, car, «en France, en Angleterre, en Allemagne, cette opinion a eu de grands hommes pour partisans ${ }^{74} »$. Il faut alors trancher, puisque le dualisme est impossible pour notre auteur anonyme, en faveur du matérialisme ou 
de l'immatérialisme, ce qui suppose une réfutation définitive de la thèse adverse. Mais cette dernière tourne court: pour l'auteur, on doit admettre ou l'existence d'un être spirituel infini ou celle d'un être matériel immensément étendu. Or, nous dit-il, la matière existe, donc Dieu n'est pas; et ainsi la thèse immatérialiste s'effondre d'elle-même. Seulement, l'existence de la matière n'est ni déduite ni prouvée, elle est tout simplement posée.

Tout cela explique peut-être le scepticisme qui semble s'abattre sur l'épistémologie de la fin du Siècle des lumières. Parce qu'irréfutable, l'idéalisme oblige les matérialistes à le déjouer sans pour autant le renverser, d'où des contournements ingénieux, comme ceux de Brissot de Warville ou de Condorcet, qui préconisent - en 1782 pour le premier, en 1785 pour le second - l'accumulation de probabilités pour parvenir à s'approcher de la vérité ${ }^{75}$, ou encore celui de Jean-Claude de La Métherie, qui, répondant au vœu exprimé par Helvétius, propose, dans ses Principes de la philosophie naturelle de 1787, un classement des certitudes dans lequel celle de l'existence des corps extérieurs se verrait attribuer la note "certain - 1», qui symbolise la quasi-certitude.

Le plus tragique, et ce sera là notre conclusion, est que tout ce déploiement d'analyse conceptuelle s'est fait à l'encontre d'un adversaire tout aussi conceptuel. L'immatérialisme de Berkeley n'est pas un «incorporalisme» déniant toute existence aux corps extérieurs, mais une philosophie qui prétend donner un statut ontologique à l'apparence sans supposer de substrat derrière les qualités sensibles que nous percevons. Rien de plus faux pour Berkeley que de réduire le monde à notre conscience, rien de plus vrai par contre que de lui refuser une existence réelle hors de toute perception spirituelle. Parce que Dieu maintient les choses dans l'être en les percevant, celles-ci acquièrent une certaine indépendance à l'égard de la perception humaine, dont elles ne sauraient dépendre. Pour avoir mal lu Berkeley, et cette erreur de lecture a ses raisons historiques, le matérialisme des Lumières a repris un problème que le siècle précédent, à travers le cartésianisme et ses critiques, lui avait légué. En tentant d'y apporter une réponse moniste, il s'est enfermé dans un monisme matériel qui, malgré tout, semblait valoir bien mieux que celui, spirituel et créé de toutes pièces, d'un opposant fantoche nommé Berkeley. L'avantage du premier sur le second est facile à cerner: postuler l'existence de la matière sans savoir pour autant ce qu'elle est équivaut à accepter l'existence d'autrui et du monde, alors que s'enfermer dans l'égoïsme revient à se penser seul existant. Ici l'égoïsme perd sa dimension métaphysique au profit d'une vision essentiellement morale, celle qui s'est peu à peu imposée au $\mathrm{XVIII}^{\mathrm{e}}$ siècle et qui consiste à faire de l'égoïste un individu centré sur son moi et indifférent à l'égard d'autrui. Au contraire, le monisme matériel est ouvert sur l'autre, et la sociabilité 
est le maître mot des Lumières face à une doctrine irréfutable conceptuellement, mais condamnable moralement. Comme l'écrivait déjà Maupertuis en réponse à la possibilité philosophique du malebranchisme, «rester seul dans l'Univers, $c^{\prime}$ est une idée bien triste ${ }^{76}{ }^{\prime}$. À travers ces quelques mots, on comprend bien comment, pour les philosophes des Lumières, une certaine idée du bonheur rendait irrecevables des conclusions immatérialistes qu'ils s'accordaient pourtant à reconnaître comme théoriquement irréfutables.

\section{SÉBASTIEN CHARLES}

\section{Notes}

1 Leibniz, Opera philosophica, édition de J. E. Erdmann, Berlin, 1840, t. I, p. 186a. Cité par Olivier Bloch dans Matière à histoires, Paris, Vrin, coll. «Histoire de la philosophie», 1997, p. 23.

2 Voir Olivier Bloch, le Matérialisme, Paris, Presses universitaires de France, coll. «Que sais-je?», 1985.

3 Sur cette réception au début $\mathrm{du}$ XVIII ${ }^{\mathrm{e}}$ siècle de l'immatérialisme, voir notre «Dos casos de recepción deformada en el siglo de las Luces: Spinoza y Berkeley», Actes du XIV Congrès interaméricain, Puebla, 2000.

4 Abraham Gaultier, Réponse en forme de dissertation à un théologien qui demande ce que veulent dire les Sceptiques, qui cherchent la verité partout dans la Nature, comme dans les écrits des Philosophes; lors qu'ils pensent que la Vie \& la Mort sont la même chose, Nyort, Jean Elies, 1714.

5 Voir Parité de la vie et de la mort. La Réponse du médecin Gaultier, textes rassemblés, présentés et commentés par Olivier Bloch, Paris-Oxford, Universitas-Voltaire Foundation, 1993. Voir aussi Matière à histoires, op. cit., p. 255-286.

6 Abraham Gaultier, Réponse en forme de dissertation, op. cit., p. 106.

7 Abraham Gaultier, Parité de la Vie $\mathcal{E}$ de la Mort, 1770, p. 72.

8 Père Tournemine, Mémoires de Trévoux, janvier 1719, p. 51. Le texte de 1718, légèrement différent, est cité par Henry M. Bracken, The Early Reception of Berkeley's Immaterialism 1713-1734, La Haye, Nijhoff, 1963 (édition révisée), p. 109.

9 Jean Meslier, Anti-Fénelon. Notes inédites sur la Démonstration de l’Existence de Dieu de Fénelon et les Réflexions sur l'athéisme Du Père Tournemine, dans CEuvres complètes, Paris, Anthropos, 1972, t. III, p. 351.

10 Jean Meslier, Mémoire de Jean Meslier, dans CEuvres complètes, Paris, Anthropos, 1972, t. III, p. 187.

11 Père Buffier, Traité des premieres véritez et de la source de nos jugemens, Paris, 1724; réédité dans le Cours de sciences sur des principes nouveaux $\mathcal{E}$ simples, Paris, Cavelier et Giffart, 1732, p. 560. 
12 Père Buffier, Élémens de métaphisique à la portée de tout le monde, Paris, 1725; réédité dans le Cours de sciences sur des principes nouveaux $\mathcal{E}$ simples, Paris, Cavelier et Giffart, 1732, p. 924. Dans une note de la traduction anglaise qu'il donne de ce texte, S. V. Bokil propose de voir en cet «écrivain écossais» Berkeley, mais il pourrait également s'agir d'Arthur Collier (voir la traduction commentée de Bokil parue dans le Indian Philosophical Quarterly, 2, 1980, p. 31).

13 Jean-Pierre de Crousaz, Examen du pyrrhonisme ancien \& moderne, La Haye, Pierre de Hondt, 1733. Même citation, à peu de choses près, dans la Logique, ou systeme de reflexions qui peuvent contribuer à la netteté $\mathcal{E}$ à l'étendue de nos Connoissances, Lausanne \& Genève, Marc-Michel Bousquet \& Comp., 1741 ( $4^{\mathrm{e}}$ édition), part. I, sect. I, chap. IV. Le passage ne se trouve pas dans la première édition de 1712 et, selon Bracken (op. cit., note de la page 3), n'apparaît qu'en 1724 dans l'édition anglaise de l'ouvrage parue à Londres.

14 François Cartaud de la Villate, Pensées critiques sur l'existence des mathématiques, Paris, Osmont \& Clousier, 1733, p. 144.

15 Jean-Robert Armogathe, Une secte fantôme au dix-huitième siècle: les Égoïstes, Paris, mémoire de maîtrise, 1970, xv/354 p. (exemplaire dactylographié, disponible à la Bibliothèque de l'École normale supérieure, cote Mémoires 1970_15). Qu'il nous soit permis de remercier ici le père Armogathe de nous avoir donné l'autorisation de lire ce travail.

16 Voir «La figure de Berkeley dans la pensée de Voltaire», à paraître dans Dix-huitième siècle, 33, 2001.

17 Saint-Hyacinthe, Recherches philosophiques sur la nécessité de s'assurer par soi-meme de la verité, Rotterdam et La Haye, Alex. Johnson, 1743, p. 95.

18 Diderot, CEuvres complètes, édition de Roger Lewinter, Paris, Le club français du livre, 1972, t. XIII, p. 902. Nous citerons l'édition Lewinter (15 tomes parus de 1969 à 1973) et, quand nous le pourrons et entre parenthèses, l'édition critique des CEuvres complètes, Paris, Hermann, en cours de publication depuis 1975, 36 vol. prévus.

19 Nous avons en effet parcouru les œuvres d'Hemsterhuis sans succès.

20 François Hemsterhuis, Lettre sur l'homme et ses rapports avec le commentaire inédit de Diderot, texte établi, présenté et annoté par Georges May, New Haven, Yale University Press, 1964, p. 10. Plus loin, Diderot condamne le recours au sens commun que Hemsterhuis, en cela disciple de Buffier, propose. Là où, pour Hemsterhuis, «la sensation de plusieurs êtres de notre espece acheve de nous convaincre de l'existence des choses hors de nous», Diderot note: «Berkeley ne serait point embarrassé de cette réponse» (p. 54). En effet, si l'existence du monde extérieur ne peut être prouvée, ce doute englobe tous les éléments de l'univers, dont les autres membres de notre espèce, qui ne sauraient alors être des témoins sûrs confirmant avec justesse nos sensations.

21 Jacques Chouillet, «Le personnage du sceptique dans les premières œuvres de Diderot», Dix-huitième siècle, 1, 1969, p. 209.

22 Jean Deprun, «Diderot devant l'idéalisme», Revue internationale de philosophie, 38 , 148-149, 1984, p. 67-78.

23 Ursula Winter, Der materialismus bei Diderot, Genève-Paris, Droz-Minard, 1972, p. 260. 
24 Alexandre Saverien, art. "Corps», dans Dictionnaire universel de Mathématique et de Physique, Paris, Rollin et Jombert, 1753, 2 t. Le passage du troisième dialogue qu'il cite (t. I, p. 225) et qui se situe selon lui aux pages 56-58 de l'édition de 1744 se trouve aux pages 205-207 de l'édition de 1750 parue à Amsterdam. H. R. Mead, dans A Bibliography of George Berkeley (Berkeley, 1910), mentionnait une édition de 1745 dont Jessop n'a pu découvrir la moindre trace (A Bibliography of George Berkeley, The Hague, Martinus Nijhoff, 1973, p. 17).

25 Bibliographie universelle ancienne et moderne, Paris, Michaud, 1817, vol. 18, p. 576.

26 En effet, le chapitre 6 du livre I de l'Essai, qui s'intéresse à la question de la perception, suit les Élémens de la philosophie de Newton (II, 7) de Voltaire paru huit ans auparavant, dans lesquels Berkeley est nommé, mais sans que sa doctrine immatérialiste soit exposée. Nous pensons, comme Merian au XVIII siècle l'affirmait déjà dans ses huit Mémoires sur le problème de Molyneux (présentés à l'Académie de Berlin entre 1770 et 1780), que Condillac n'a connu Berkeley que de seconde main. Merian écrivait ainsi, dans le $\S 3$ du sixième Mémoire de 1777: «si j'ose dire librement ma pensée, je croirois plutôt que ni l'un [Condillac] ni l'autre [Diderot] de ces philosophes n'ont lu l'ouvrage du Docteur, quoi qu'il ait paru dès l'année 1709 , \& qu'il ait été traduit en françois [en 1734, à la suite de l'Alciphron]. [...] On diroit qu'ils ignorent jusqu'au titre de cet ouvrage: Mr l'Abbé de Condillac se méprend sur le nom même de l'auteur, qu'il écrit Barclai au lieu de Berkeley. Ils ne le citent jamais, \& ne rapportent sa doctrine que d'après des morceaux que Voltaire a insérés dans sa Philosophie de Newton» (361).

27 Voir Arthur M. Wilson, Diderot. Sa vie et son auvre, Paris, Laffont/Ramsay, coll. «Bouquins», 1985, p. 65-68.

28 Notamment les articles «Abstraction», «Body», «External» et "Matter» parus dans la Cyclopxdia or an Universal Dictionary of the Arts $\mathcal{E}$ Sciences à Londres en 1728.

29 Faut-il dès lors s'étonner si le premier texte de Rousseau qui mentionne l'immatérialisme (le Discours sur les sciences et les arts) date de 1750? Quant à D'Alembert, il ne pouvait manquer de connaître Berkeley à cette période, puisqu'il avait lu la Lettre sur les aveugles pour rédiger l'article «Aveugle» du tome I de l'Encyclopédie. Deux lettres de Turgot, qui seront publiées de manière posthume, datent de cette période et concernent Berkeley. Sur Rousseau et Berkeley, voir notre «De Pyrrhon à Berkeley: Rousseau et le scepticisme», à paraître dans la Revue de métaphysique et de morale; et sur Turgot et Berkeley, voir notre «Turgot ou la pensée fragmentée. Étude d'une critique de l'immatérialisme berkeleyen", Études maritainiennes, 15, 1999, p. 157-167.

30 Condillac, Essai sur l'origine des connaissances humaines, I, I, 1, Paris, Éditions Alive, 1998, p. 31

31 Diderot, Promenade du sceptique, dans O. C., 1969, t. I, p. 358 (tome II, p. 118).

32 Diderot, Lettre sur les aveugles à l'usage de ceux qui voient, dans O. C., 1969, t. II, p. 192 (t. IV, p. 44).

33 Voir notre «L'abbé Raynal, Author of an Unnoticed Review of Berkeley's Dialogues", Berkeley Newsletter, 15, 1997-1998, p. 14-18.

34 D’Alembert, art. «Égoïstes», dans Encyclopédie, Paris, 1755, t. V, p. 431.

35 D’Alembert, art. «Corps», dans Encyclopédie, Paris, 1754, t. IV, p. 261.

36 Diderot, art. «Pyrrhonienne ou Sceptique», dans Encyclopédie, Paris, 1765, t. XIII, p. 614. Cette idée est plagiée par Delisle de Sales dans De la philosophie de la nature, 
ou Traité de morale pour le genre humain tiré de la Philosophie et fondé sur la nature, Londres, 1789 ( $5^{\mathrm{e}}$ édition), t. I, p. 25.

37 Diderot, lettre à Vialley de juillet 1766, dans Correspondance, édition de Georges Roth et Jean Varloot, Paris, Éditions de Minuit, 1961, t. VI, p. 231-232.

38 Diderot, lettre à Falconet du 29 décembre 1766, dans ibid., t. VI, p. 376.

39 Diderot, le Rêve de D'Alembert, dans O. C., 1971, t. VIII, p. 71 (t. XVII, p. 108).

40 Diderot, Essai sur les règnes de Claude et de Néron, dans O. C., t. XIII, p. 501 (t. XXV, p. 291).

41 Le Guay de Prémontval, le Diogène de D'Alembert, ou Diogène décent, Berlin, Schneider, 1755, p. 154-155.

42 Diderot, art. «Pyrrhonienne ou Sceptique», Encyclopédie, Paris, 1765, t. XIII, p. 614.

43 Diderot, CEuvres complètes, éditées par Jules Assézat et Maurice Tourneux, Paris, Garnier, 1875, t. I, note 1 de la page 119.

44 Diderot, art. «Pyrrhonienne ou Sceptique», Encyclopédie, Paris, 1765, t. XIII, p. 614.

45 Louis de Beausobre, le Pirrhonisme du sage, Berlin, 1754, p. 23: «Pourrions-nous démontrer plus facilement l'erreur des Égoistes, \& des Matérialistes? Il y a sans doute de la folie à s'imaginer qu'on est le seul être existant, \& l'on humilie peut-être l'homme, en donnant à la matière la force de penser; mais je ne puis démontrer qu'une folie, \& qu'une idée humiliante soient des erreurs.»

46 Ibid., p. 103.

47 L'attribution de cet article à Diderot n'est pas formellement établie. Sur ces problèmes d'attribution, voir Jacques Proust, Diderot et l'Encyclopédie, Paris, Albin Michel, coll. «Bibliothèque de l'évolution de l'humanité», 1995.

48 Art. «Doute», Encyclopédie, Paris, 1755, t. V, p. 89.

49 Huet, Traité philosophique de la foiblesse de l'entendement humain, Amsterdam, Henri du Sauzet, 1723, p. 242-243.

50 Art. «Doute», Encyclopédie, Paris, 1755, t. V, p. 89.

51 Helvétius, De l'Esprit, discours premier, chap. I, note, dans CEuvres complètes d'Helvétius, Paris, P. Didot, 1795, t. I, p. 201-202.

52 Ibid., p. 202.

53 Ibid., p. 202-203.

54 Voir La Métherie, Principes de la philosophie naturelle, dans lesquels on cherche à déterminer les degrés de certitude ou de probabilité des connoissances humaines, Genève, $1787,2 \mathrm{t}$.

55 Barruel, les Helviennes, ou Lettres provinciales philosophiques (1784), Paris, De Méquignon et Boiste, 1823 ( $6^{\mathrm{e}}$ édition), t. II, p. 182.

56 D’Holbach, Essai sur les préjugés, ou De l'influence des opinions sur les mœurs $\mathcal{E}$ sur le bonheur des hommes, dans CEuvres philosophiques complètes, Paris, Éditions Alive, 1999, t. II, p. 116.

57 Ferdinando Galiani et Louise d'Épinay, Correspondance. I. 1769-1770, texte établi par Daniel Maggetti et annoté par Daniel Maggetti en collaboration avec Georges Dulac, Paris, Desjonquères, coll. «XVIII siècle», 1992, p. 196. 
58 Yvon Belaval, introduction aux CEuvres complètes d'Helvétius, Hildesheim, Olms, 1967-1969, t. I, p. XV-XVI.

59 Dom Deschamps, la Vérité ou le vrai système, cité par Paul Vernière, Spinoza et la pensée française, Paris, Presses universitaires de France, 1954, p. 631.

60 Pierre Naville, D'Holbach et la philosophie scientifique au XVIII siècle, Paris, Gallimard, coll. «Bibliothèque des idées», 1967, p. 228.

61 D'Holbach, Système de la nature, $2^{\mathrm{e}}$ partie, chap. IV, dans CEuvres philosophiques complètes, t. II, p. 455: «S'il n'y a rien hors d'un être qui existe nécessairement, il faut qu'il soit unique.»

62 Ibid.

63 Ibid., $1^{\text {re }}$ partie, chap. X, t. II, p. 261.

64 Ce rapprochement a été vu également par Roland Mortier dans «Holbach et Diderot: affinités et divergences", Revue de l'Université de Bruxelles, 2-3, 1972, p. 231.

65 D'Holbach, op. cit., note de la p. 261.

66 Ibid., p. 261.

67 D'Holbach, la Morale universelle ou les devoirs de l'homme fondés sur sa nature, Amsterdam, M.-M. Rey, 1776, p. XII.

68 Ibid., p. XII-XIII.

69 Nous remercions Miguel Benítez de nous avoir fourni une copie du seul manuscrit existant (Rouen, B. M., M 74). Nous indiquerons en chiffres romains les chapitres du traité et les ferons suivre du numéro du feuillet.

70 Jordanus Brunus Redivivus, ou Traité des Erreurs populaires, Ouvrage critique, historique et philosophique, imité de Pomponace, II, f. 67.

71 lbid., V, f. 206.

72 Ibid., III, f. 121.

73 Ibid., III, f. 122.

74 Ibid., III, f. 123.

75 Pour Brissot de Warville, voir son De la vérité ou Méditation sur les moyens de parvenir à la vérité dans toutes les connoissances humaines, Neuchâtel, 1782.

Concernant Condorcet, voir son Essai sur l'application de l'analyse à la probabilité des décisions rendues à la pluralité des voix, Paris, 1785. Voir aussi Richard H. Popkin, «Brissot and Condorcet: Skeptical Philosophers», dans J. Van der Zande et R. H. Popkin (édit.), The Skeptical Tradition around 1800. Skepticism in Philosophy, Science and Society, Dordrecht, Kluwer, 1998, p. 31-39.

76 Maupertuis, Lettre IV, dans CEuvres de Maupertuis, Lyon, Jean-Marie Bruyset, 1768, t. II, p. 236. 